\title{
EXTRAPOLATION OF SEQUENCES USING A GENERALIZED EPSILON-ALGORITHM
}

\author{
MICHAEL N. BARBER AND C. J. HAMER
}

(Received 24 September 1980)

(Revised 24 May 1981)

\begin{abstract}
The problem of estimating the limit $f_{\infty}$ of a sequence $f_{n}$ converging as $f_{n}-f_{\infty}=O\left(n^{-\lambda}\right)$ as $n \rightarrow \infty$, where $\lambda>0$, is discussed. Using the generalization of the $\varepsilon$-algorithm proposed recently by Vanden Broeck and Schwartz, an acceleration scheme is developed. The method is illustrated on several test sequences and compared to other acceleration procedures.
\end{abstract}

\section{Introduction}

The problem of estimating the limit of a sequence given a finite number of terms is a common one in applied mathematics and theoretical physics. Various procedures for doing so have been devised (for reviews, see [2] and [11]). The applicability of these methods depends crucially on the way the limit is approached.

Recently Vanden Broeck and Schwartz [12] have introduced a one-parameter family of non-linear sequence transformations defined by the relations

$$
\begin{aligned}
\varepsilon_{n}^{(m)} & =\alpha \varepsilon_{n}^{(m-1)}+1 /\left(f_{n+1}^{(m)}-f_{n}^{(m)}\right), \\
f_{n}^{(m+1)} & =f_{n}^{(m)}+1 /\left(\varepsilon_{n}^{(m)}-\varepsilon_{n-1}^{(m)}\right) .
\end{aligned}
$$

Here $\alpha$ is an arbitrary free parameter, $\varepsilon_{n}^{(-1)} \equiv 0$ and $f_{n}^{(0)}, n=1,2, \ldots, N$, are the available terms of the original sequence whose limit is to be estimated. One iteration of (1), namely $f_{n}^{(1)}$, is equivalent to the Shanks transform [10], (or the 
Aitken-algorithm [9], p. 348) which is then iterated by setting $\alpha=0$. If $\alpha=1$, (1) reduces to Wynn's $\varepsilon$-algorithm [13] for generating the diagonal Padé approximants to a power series. In this case, $f_{n}^{(0)}$ is the $n$th partial sum of the original series. We shall refer to the transformation (1) as the "generalized $\varepsilon$-algorithm".

Like the Shanks transform, (1) is exact for sequences of the form

$$
f_{n}=f_{\infty}+A q^{n}
$$

in the sense that $f_{n}^{(1)}=f_{\infty}$. Thus (1) is likely to successfully accelerate the convergence of sequences converging linearly, that is those asymptotically satisfying

$$
f_{n}-f_{\infty} \sim q\left(f_{n-1}-f_{\infty}\right) \text { as } n \rightarrow \infty .
$$

By judicious choice of the parameter $\alpha$, Vanden Broeck and Schwartz were able to successfully extrapolate a far wider variety of sequences including formally divergent ones. Despite these spectacular examples, little mathematical justification of the procedure is available. Nor is it clear what value of $\alpha$ should be chosen for "optimal" acceleration of any particular class of sequences.

In this paper, we specifically consider the extrapolation of sequences converging logarithmically, that is, as

$$
f_{n}-f_{\infty}=O\left(n^{-\lambda}\right) \text { as } n \rightarrow \infty,
$$

where $\lambda>0$. In particular, in Section 2, we prove that if the parameter $\alpha$ in (1) is chosen to be -1 , then $f_{n}^{(2)}$ converges to $f_{\infty}$ at least as $o\left(n^{-\lambda}\right)$ and possibly as fast as $O\left(n^{-\lambda-2}\right)$. Secondly, we show that if the generating function,

$$
F(x)=\sum_{n=0}^{\infty} f_{n} x^{n}
$$

of the sequence $\left\{f_{n}\right\}_{n=0}^{\infty}$ is exactly of the form

$$
F(x)=f_{\infty}(1-x)^{-1}+A(1-x)^{-1+\lambda} \text { where } \lambda>0,
$$

then $f_{n}^{(2)}$ is exactly $f_{\infty}=\lim _{n \rightarrow \infty} f_{n}$ if $\alpha$ is again chosen to be -1 .

On the basis of these results, we formulate in Section 3 a new algorithm to accelerate logarithmically convergent sequences. This algorithm is then tested and compared to alternative algorithms using several test sequences.

\section{Basic theorems}

Our application of the generalized $\varepsilon$-algorithm to the extrapolation of logarithmically convergent sequences is based on the following theorems. 
THEOREM 1. Let the sequence $\left\{f_{n}\right\}$ approach its limit $f_{\infty}$ as

$$
f_{n}-f_{\infty}=A n^{-\lambda}+o\left(n^{-\lambda}\right) \text { as } n \rightarrow \infty,
$$

where $\lambda>0$. Then if $f_{n}^{(2)}(\alpha)$ is the second iterate of $f_{n}$ under the generalized $\varepsilon$-algorithm (1) for arbitrary $\alpha$,

$$
f_{n}^{(2)}(-1)-f_{\infty}=o\left(n^{-\lambda}\right)
$$

Proof. We first observe from (1) that if $g_{n} \equiv \mu f_{n}+\eta$, where $\mu$ and $\eta$ are constants, then $g_{n}^{(m)}=\mu f_{n}^{(m)}+\eta$. Consequently we may take $f_{\infty}=0$ and $A=1$ without loss of generality.

Thus we set

$$
f_{n}^{(0)}=n^{-\lambda}+o\left(n^{-\lambda}\right) \text { as } n \rightarrow \infty .
$$

Explicit calculation gives

$$
\varepsilon_{n}^{(0)}=-\lambda^{-1} n^{1+\lambda}+o\left(n^{1+\lambda}\right)
$$

so that

$$
f_{n}^{(1)}=n^{-\lambda} /(1+\lambda)+o\left(n^{-\lambda}\right) \text { as } n \rightarrow \infty .
$$

Repeating the calculation but allowing for an arbitrary value of the parameter $\alpha$ which enters the definition of $\varepsilon_{n}^{(1)}$ gives

$$
\varepsilon_{n}^{(1)}=-(\alpha+1+\lambda) \lambda^{-1} n^{1+\lambda}+o\left(n^{1+\lambda}\right),
$$

so that

$$
f_{n}^{(2)}(\alpha)=(1+\alpha) n^{-\lambda} /((1+\lambda)(1+\alpha+\lambda))+o\left(n^{-\lambda}\right)
$$

The result (8) is now immediate on observing that the leading $n$ dependence of $O\left(n^{-\lambda}\right)$ vanishes if $\alpha$ is set to -1 .

A more detailed consideration of correction terms yields the stronger result contained in Theorem 2.

THEOREM 2. Let $\left\{f_{n}\right\}$ approach its limit $f_{\infty}$ as

$$
f_{n}-f_{\infty}=A n^{-\lambda}\left[1+b n^{-\Delta}+o\left(n^{-\Delta}\right)\right] \text { as } n \rightarrow \infty \text {, }
$$

then

$$
f_{n}^{(2)}(-1)-f_{\infty}=A n^{-\lambda} \rho_{n}
$$

where

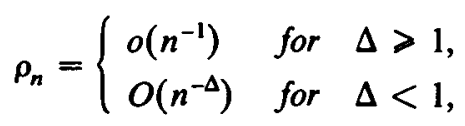

as $n \rightarrow \infty$. 
Proof. Again we set $f_{\infty}=0, A=1$ and write

$$
f_{n}^{(0)}=n^{-\lambda}\left[1+E_{n}^{(0)}\right] \text {, }
$$

where

$$
E_{n}^{(0)}=b n^{-\Delta}+o\left(n^{-\Delta}\right) \text { as } n \rightarrow \infty .
$$

From (1a) we then calculate $\varepsilon_{n}^{(0)}$ which we write as

$$
\varepsilon_{n}^{(0)}=-\lambda^{-i} n^{i+\lambda}\left[1+F_{n}^{(0)}\right] \text {, }
$$

where

$$
F_{n}^{(0)}=\phi_{n}^{(0)} /\left[1-\phi_{n}^{(0)}\right]
$$

with

$$
\begin{aligned}
\phi_{n}^{(0)}=(n / \lambda)\left\{\left[(1+1 / n)^{-\lambda}-1+\lambda / n\right]+(1\right. & +1 / n)^{-\lambda}\left[E_{n+1}^{(0)}-E_{n}^{(0)}\right] \\
& \left.+E_{n}^{(0)}\left[(1+1 / n)^{-\lambda}-1\right]\right\} .
\end{aligned}
$$

For future reference, we note that if $E_{n}^{(0)}$ satisfies (18), then $\phi_{n}^{(0)}$ and hence $F_{n}^{(0)}$ are $O\left(n^{-\Delta}\right)$ if $\Delta<1$, but $O\left(n^{-1}\right)$ if $\Delta \geqslant 1$.

The calculation of $f_{n}^{(1)}, \varepsilon_{n}^{(1)}$ and finally $f_{n}^{(2)}(-1)$ is now straightforward if somewhat tedious. The resulting expressions are most conveniently written as

$$
\begin{aligned}
& f_{n}^{(1)}=(1+\lambda)^{-1} n^{-\lambda}\left[1+E_{n}^{(1)}\right], \\
& \varepsilon_{n}^{(1)}=-n^{1+\lambda}\left[1+F_{n}^{(1)}\right],
\end{aligned}
$$

and finally

$$
f_{n}^{(2)}(-1)=n^{-\lambda} \rho_{n}
$$

where

$$
\begin{aligned}
& E_{n}^{(1)}=(1+\lambda) E_{n}^{(0)}-\lambda \psi_{n}^{(0)} /\left(1-\psi_{n}^{(0)}\right), \\
& F_{n}^{(1)}=-\lambda^{-1} F_{n}^{(0)}+(1+\lambda) \lambda^{-1} \phi_{n}^{(1)} /\left(1-\phi_{n}^{(1)}\right)
\end{aligned}
$$

and

$$
\rho_{n}=(1+\lambda)^{-1}\left[E_{n}^{(1)}-\psi_{n}^{(1)} /\left(1-\psi_{n}^{(1)}\right)\right]
$$

In (25)-(27), $\phi_{n}^{(1)}$ is given by (21) with the $E^{(0)}$ 's replaced by $E^{(1)}$ 's and

$$
\begin{aligned}
\psi_{n}^{(i)}=(n /(1+\lambda))\left\{(1-1 / n)^{1+\lambda}\right. & {\left[F_{n-1}^{(i)}-F_{n}^{(i)}\right] } \\
-F_{n}^{(i)}\left[1-(1-1 / n)^{1+\lambda}\right] & {\left.\left[(1-1 / n)^{1+\lambda}-1+((1+\lambda) / n)\right]\right\} } \\
+ & \text { for } i=0,1 .
\end{aligned}
$$


It remains now to estimate the order of $\rho_{n}$. In view of the remark after (21) we consider three cases.

Case I: $\Delta<1$. In this case, $\phi_{n}^{(0)}=O\left(n^{-\Delta}\right)$ and a straightforward sequence of calculations establishes that $E_{n}^{(1)}, F_{n}^{(0)}, F_{n}^{(1)}$ and $\rho_{n}$ are all similarly $O\left(n^{-\Delta}\right)$, with explicitly,

$$
\rho_{n}=\left(\left(\Delta^{2}(1-\Delta)^{2} b\right) /\left(\lambda^{2}(1+\lambda)^{2}\right)\right) n^{-\Delta}+o\left(n^{-\Delta}\right)
$$

which establishes (16b).

Case II: $\Delta>1$. In this case, the dominant term in $\phi_{n}^{(0)}$ is the first, which yields

$$
\phi_{n}^{(0)}=\frac{1}{2}(\lambda+1) n^{-1}+o\left(n^{-1}\right) \text {. }
$$

However, the leading $O\left(n^{-1}\right)$ term drops out of $E_{n}^{(1)}$ and $\psi_{n}^{(1)}$ leaving both of $o\left(n^{-1}\right)$. Hence $\rho_{n}$ is also $o\left(n^{-1}\right)$.

Case III: $\Delta=1$. In this case, all three terms in (21) are of the same order leading to

$$
\phi_{n}^{(0)}=\frac{1}{2}(\lambda+1)(1-2 b / \lambda) / n+o\left(n^{-1}\right) .
$$

However, the leading $O\left(n^{-1}\right)$ is again removed leaving $\rho_{n}=o\left(n^{-1}\right)$ which establishes (16a) and completes the proof of the theorem.

The exact order of $\rho_{n}$ in (15) for $\Delta \geqslant 1$ depends on the nature of subsequent correction terms in (14). We do not present a general analysis, but note that if, as occurs often in practical examples,

$$
f_{n}-f_{\infty} \sim A n^{-\lambda}\left[1+\sum_{j=1}^{\infty} b_{j} n^{-j}\right] \text { as } n \rightarrow \dot{\infty},
$$

then a tedious asymptotic analysis establishes that

$$
f_{n}^{(2)}-f_{\infty} \sim A^{\prime} n^{-\lambda-2} \text { as } n \rightarrow \infty
$$

where $A^{\prime}$ is non-zero.

In assessing the applicability of a sequence extrapolation algorithm it is useful to know of the cases for which the algorithm is exact. We have already noted in the Introduction that $f_{n}^{(1)}$ is exactly the limit of a sequence of the form $f_{n}^{(0)}=f_{\infty}+q^{n}$. The following theorem gives a further example.

THEOREM 3. Let the generating function of the sequence $\left\{f_{n}\right\}, F(x)=\sum_{n=0}^{\infty} x^{n} f_{n}$, be of the form

$$
F(x)=f_{\infty}(1-x)^{-1}\left[1+A(1-x)^{\lambda}\right] .
$$

Then with $\alpha=-1, f_{n}^{(2)}(-1)$ is exactly $f_{\infty}=\lim _{n \rightarrow \infty} f_{n}$. 
Proof. We note first that if $F(x)$ is of the form (34), then

$$
f_{n} \simeq f_{\infty}\left[1+A n^{-\lambda}+O\left(n^{-1-\lambda}\right)\right] \text { as } n \rightarrow \infty,
$$

so that the remark after Theorem 2 implies that

$$
f_{n}^{(2)}(-1)=f_{\infty}+O\left(n^{-2-\lambda}\right) .
$$

We now show that $f_{n}^{(2)}(-1)$ is exactly $f_{\infty}$.

To do so, we expand (34) and write

$$
f_{n}=f_{\infty}+C_{n},
$$

where $C_{n}$ satisfies the recurrence relation

$$
C_{n}=(n-\lambda) C_{n-1} / n \text { with } C_{0}=A f_{\infty} .
$$

Hence

$$
\varepsilon_{n}^{(0)}=-(n+1) /\left(\lambda C_{n}\right)
$$

and

$$
f_{n}^{(1)}=f_{\infty}+C_{n} /(1+\lambda) .
$$

Repeating the calculation but allowing for the factor of $\alpha$ gives

$$
\varepsilon_{n}^{(1)}=-(n+1)(\alpha+\lambda+1) /\left(\lambda C_{n}\right),
$$

so that

$$
f_{n}^{(2)}=f_{\infty}+C_{n}(\alpha+1) /((\lambda+1)(\alpha+\lambda+1)),
$$

from which the theorem follows immediately on putting $\alpha=-1$.

\section{Numerical examples}

The result of Theorem 2 suggests that further acceleration can be achieved by re-applying the transformation to $f_{n}^{(2)}$. In doing so, the parameter $\alpha$ entering $\varepsilon_{n}^{(2)}$ must be reset to zero to offset effects arising from $\varepsilon_{n}^{(1)}$. Thus the successive transformations are generated by the relations

$$
\left.\begin{array}{r}
f_{n}^{(m+1)}=f_{n}^{(m)}+1 /\left[\varepsilon_{n}^{(m)}-\varepsilon_{n-1}^{(m)}\right] \\
\varepsilon_{n}^{(m)}=\alpha_{m} \varepsilon_{n}^{(m-1)}+1 /\left[f_{n+1}^{(m)}-f_{n}^{(m)}\right]
\end{array}\right\}
$$

where

$$
\alpha_{m}=-\left[1-(-)^{m}\right] / 2
$$

We shall refer to this transformation as the alternating e-algorithm. One' should also note that on each iteration, that is from $f_{n}^{(2 m)}$ to $f_{n}^{(2 m+2)}$, four terms of the sequence are lost. Thus in practice the number of possible iterations is usually very limited. 
TABLE I. Logarithmically convergent test series (see [11])

\begin{tabular}{|c|c|c|}
\hline No. & $n$th term & Sum \\
\hline 1 & $1 / n^{2}$ & $\pi^{2} / 6=1.644934066848 \ldots$ \\
2 & $\frac{1+n^{2}+n^{4}}{n^{2}\left(1+n^{4}\right)}$ & $2.233411647652 \ldots$ \\
3 & $\frac{2 n-1}{n(n+1)(n+2)}$ & $3 / 4$ \\
4 & $\sin (1 / n) \ln (\cos (1 / \sqrt{n}))$ & $-0.852090754199 \ldots$ \\
5 & $(1 / n)+\ln (1-1 / n)$ & Euler's constant $=0.577215664 \ldots$ \\
6 & $\left(a_{1}=1\right)$ & $(\ln 2)(\ln K)=0.68472478856 \ldots$ \\
7 & $\ln \left(\frac{n+1}{n}\right) \ln \left(\frac{n+2}{n+1}\right)$ & where $K=$ Khinchin's constant. \\
8 & $\left(n+e^{1 / n}\right)^{-\sqrt{2}}$ & $1.713796735540 \ldots$ \\
& $(-1 / 2) \frac{(-)^{n+1}}{n-1}$ & {$[\Gamma(1 / 4)]^{2}$} \\
\hline
\end{tabular}

Algorithms for the acceleration of logarithmically convergent sequences have been reviewed and compared by Smith and Ford [11]. Their numerical testing was performed on the eight sequences $S_{n}=\sum_{j=1}^{n} a_{j}$ listed in Table I. On the basis of these results they concluded that the Levin $u$-transform [6] defined by

$$
T_{k n}=\mathscr{D}\left(\left\{S_{j}\right\}\right) / \mathscr{D}(\{1\})
$$

where

$$
\mathcal{D}\left(\left\{S_{j}\right\}\right)=\left|\begin{array}{ccccc}
S_{n} & S_{n+1} & S_{n+2} & \cdots & S_{n+k} \\
R_{n} & R_{n+1} & \cdots & & R_{n+k} \\
\frac{R_{n}}{n} & \frac{R_{n+1}}{n+1} & \cdots & & \frac{R_{n+k}}{n+k} \\
\cdot & \vdots & & \vdots \\
\frac{R_{n}}{n^{k-1}} & \frac{R_{n+1}}{(n+1)^{k-1}} & \cdots & & \frac{R_{n+k}}{(n+k)^{k-1}}
\end{array}\right|,
$$

with

$$
R_{n}=n a_{n}=n\left(S_{n}-S_{n-1}\right)
$$

was the most efficient.

A close second was the $\theta$-algorithm of Brezinski [1] defined by the equations:

$$
\begin{gathered}
\theta_{n}^{(-1)}=0, \quad \theta_{n}^{(0)}=S_{n}, \\
\theta_{n}^{(2 k+1)}=\theta_{n}^{(2 k-1)}+1 / \Delta \theta_{n}^{(2 k)}, \\
\theta_{n}^{(2 k+2)}=\theta_{n+1}^{(2 k)}+\Delta \theta_{n+1}^{(2 k)} \Delta \theta_{n+1}^{(2 k+1)} / \Delta^{2} \theta_{n}^{(2 k+1)},
\end{gathered}
$$


where $\Delta \theta_{n}=\theta_{n+1}-\theta_{n}$. For $k=0$, this corresponds to the $W$-transform of Lubkin [7], which is of special interest here since its properties are similar to those of the alternating $\varepsilon$-algorithm (43). In particular, Drummond [4] has shown that if

$$
S_{n}=S_{\infty}+A n^{-\lambda}(1+O(1 / n))
$$

then

$$
\theta_{n}^{(2)}=S_{\infty}+O\left(n^{-\lambda-2}\right),
$$

while the exactness of $\theta_{n}^{(2)}$ on sequences satisfying the conditions of Theorem 3 follows from the results of Cordellier [3] (see also [11]). Since in transforming from $\theta_{n}^{(0)}=S_{n}$ to $\theta_{n}^{(2)}$ only three terms are lost compared to four for the alternating $\varepsilon$-algorithm, it would appear that the $\theta$-algorithm should be a more efficient accelerator*.

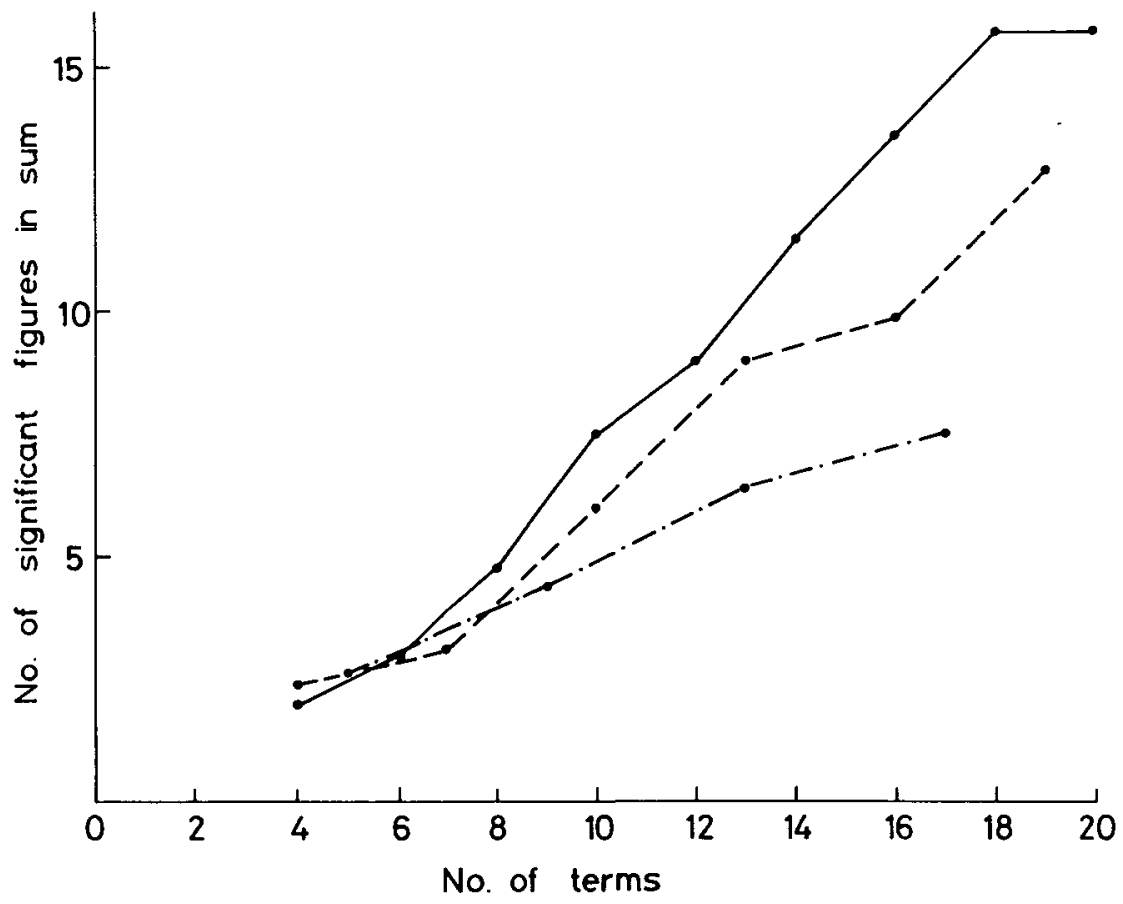

Figure 1. Performance of alternating $\varepsilon$-algorithm (-·-•-), $\theta$-algorithm (----) and Levin $u$-transform (-), averaged over the series in Table I.

-Drummond [4] has devised an acceleration procedure which also satisfies (48) and (49) but at the cost of only two terms. This method however requires knowledge of $\lambda$ which in many situations is not available. 
This expectation is borne out for the series given in Table I. Following Smith and Ford [11], we summarize these tests by plotting in Figure 1, the number of significant digits of the limit as a function of the number of terms used. All calculations were performed in double precision on a CYBER 72 using FORTRAN. The superior performance of the Levin $u$-transform is evident, while the alternating $\varepsilon$-algorithm is a very poor third. Indeed on series ( 7$)$, for which both the $\theta$-algorithm and the $u$-transform gave better than 10 figures from 20 terms, the alternating $\varepsilon$-algorithm gave only three.

Closer inspection of the sums in Table I shows that this is not in fact, a very sensitive or wide ranging test as only (7) and (8) do not converge as $1 / n$. Theorem 3 suggests that one should also compare the algorithms on sequences whose generating functions have the form

$$
F(x) \sim(1-x)^{-1}\left[1+\sum_{i} \alpha_{i}(1-x)^{-\gamma_{i}}\right] \text { as } x \rightarrow 1 .
$$

This we have done using the generating functions listed in Table II. The results, again presented as a significance plot, are indicated by Figure 2. Rather suprisingly, the alternating $\varepsilon$-algorithm (43) is at least as efficient as the $\theta$-algorithm, with the Levin $u$-transform significantly less so. Two other trends should be mentioned. The accuracy of both the $\theta$ - and alternating $\varepsilon$-algorithms deteriorates if the number of algebraic singularities increases, for example 20 terms

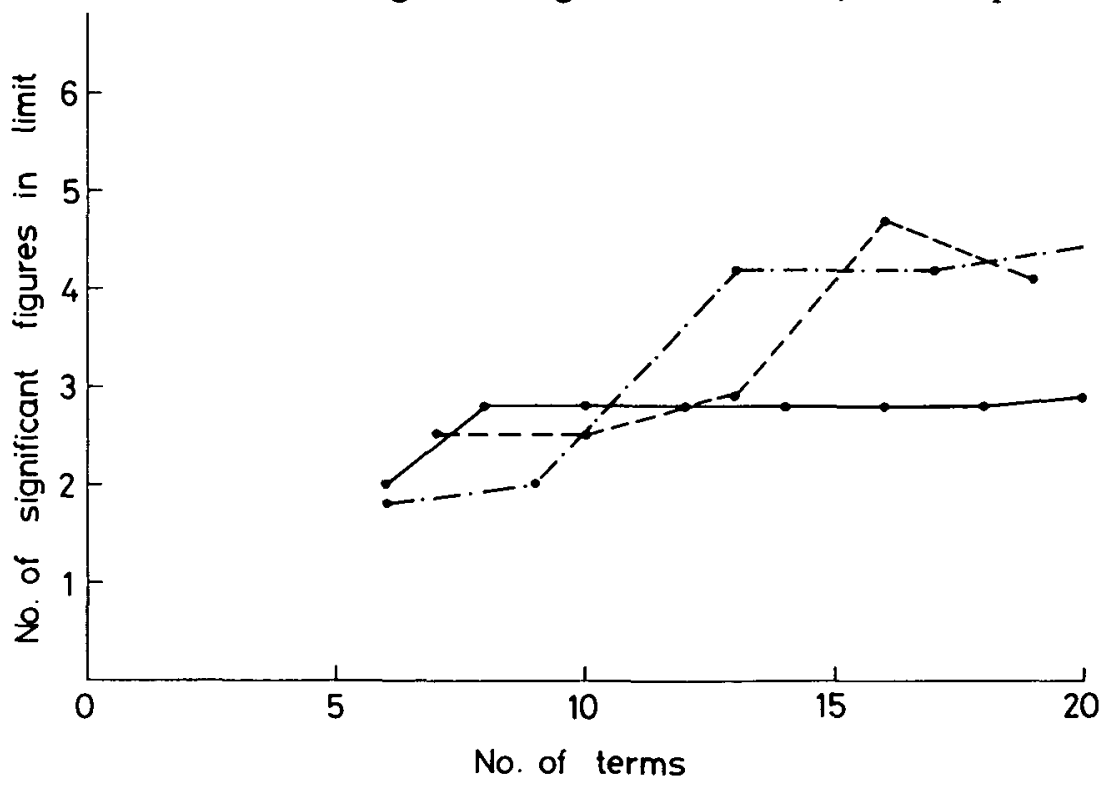

Figure 2. Performance of alternating e-algorithm (-·-·- ), $\theta$-algorithm (----) and $u$-transform (-), averaged over the sequences generated by the functions in Table II. 
TABLE II. Test generating functions

\begin{tabular}{|c|c|}
\hline No. & Function \\
\hline 1 & $(1-x)^{-1}+(1-x)^{1 / 2}+(1-x)^{-1 / 4}$ \\
2 & $(1-x)^{-1}+(1-x)^{-3 / 4}+(1-x)^{-1 / 2}$ \\
3 & $(1-x)^{-1}+(1-x)^{-0.6}+(1-x)^{-0.5}$ \\
4 & $(1-x)^{-1}+(1-x)^{-1 / 4}+(1-x)^{-1 / 3}$ \\
5 & $(1-x)^{-1}+(1-x)^{-1 / 4}+(1-x)^{-1 / 2}+(1-x)^{-3 / 4}$ \\
6 & $(1-x)^{-1}+(1-x)^{-0.2}+(1-x)^{-0.4}+(!-x)^{-0.6}$ \\
\hline
\end{tabular}

of the sequence (1) of Table II yields 5 figure accuracy by both methods, while 20 terms of sequence (5) gives only 4 figure accuracy by the alternating $\varepsilon$-algorithm and 2 by the $\theta$-algorithm. Secondly, if one of the exponents $\gamma_{i}$ in (50) is close to unity, corresponding to a small value of $\lambda$ in (48), convergence is again slow. Indeed, if $\lambda \lesssim 0.25$ we have found it almost impossible to extract more than 2 figure accuracy from 20 terms with any algorithm.

Our interest in sequences generated by functions of the form (50) arose from extrapolation problems in statistical mechanics. Our final example on the alternating $\varepsilon$-algorithm and its comparison with the $\theta$-algorithm is drawn specifically from this area [5]. The first column of Table III lists the first seven terms of a sequence ${ }^{* *} \chi_{n}$ which is expected to diverge as $n \rightarrow \infty$ as

$$
\chi_{n} \sim A n^{\omega}\left[1+O\left(n^{-\Delta}\right)\right]
$$

where probably $\Delta \geqslant \frac{1}{2}$. The problem is to estimate $\omega$. To do so, we define

$$
\rho_{n}=n\left(\chi_{n+1}-\chi_{n}\right) / \chi_{n},
$$

which, given (51), approaches $\omega$ as

$$
\rho_{n}=\omega+O\left(n^{-\Delta}\right) \text { for } n \rightarrow \infty
$$

Thus the sequence $\rho_{n}$ tabulated in the second column of Table III is precisely of the form analysed in this paper. The third column of Table III lists $\rho_{n}^{(2)}$, the $\varepsilon$-algorithm transform; further iterations not being feasible because of the shortness of the series. The extrapolation of $\rho_{n}$ by the $\theta$-algorithm is shown in

* Specifically, $\chi_{n}$ is the Hamiltonian field theoretic analog of the susceptibility of the 2-dimensional 3-state Potts (or $Z_{3}$-model) on a chain of $(n+1)$-sites. The exponent $\omega$ is then related to the nature of the critical divergence of the susceptibility of the infinite chain [5]. 
TABLE III. Extrapolation of $\left\{\rho_{n}\right\}$ (see text)

\begin{tabular}{|l|l|c|c|c|}
\hline$n$ & $x_{n}$ & $\rho_{n}=n\left[x_{n+1}-x_{n}\right] / x_{n}$ & $\rho_{n}^{(2)}$ & $\theta_{n}^{(2)}$ \\
\hline 1 & 2.821367 & 1.106499 & & 1.742861 \\
2 & 5.943208 & 1.343916 & & 1.738696 \\
3 & 9.936795 & 1.449853 & 1.733512 & 1.737326 \\
4 & 14.739091 & 1.510249 & 1.734886 & \\
5 & 20.304015 & 1.549378 & & \\
6 & 26.595732 & 1.576828 & & \\
7 & 33.585213 & & & \\
\hline
\end{tabular}

the last column of Table III. In this example, both methods have similar accuracy and together give confidence to the estimate

$$
\lim _{n \rightarrow \infty} \rho_{n}=\omega=1.734 \pm 0.002,
$$

which agrees very favourably with the theoretically expected value ([5], [8]) of

$$
\omega=26 / 15=1.73333 \ldots
$$

One final point should be made concerning round-off. For the numerical examples given in Tables I and II, all sequence terms were computed to machine accuracy (28 figures on a CYBER 72 in double precision). Running the program in single precision (14 figures) approximately halved the accuracy obtainable, although interestingly the effect on the alternating $\varepsilon$-algorithm was less than on either $\theta$ or Levin $u$-transforms. In our experience, the Levin $u$-transform, while very accurate on series of the type in Table $I$, is somewhat subject to round-off. The "real" series of Table III is, on the other hand, known only to the accuracy quoted. Here round-off is presumably more serious. However, the problem is limited by the small number of iterations performed.

\section{Conclusion}

In this paper, we have developed a new method for accelerating the convergence of sequences converging as

$$
f_{n}=f_{\infty}+O\left(n^{-\lambda}\right) \text {. }
$$

The method makes use of the generalized $\varepsilon$-algorithm (1) introduced recently by Vanden Broeck and Schwartz [12]. In essence, it consists of using their algorithm alternately with $\alpha=0$ and $\alpha=-1$ to generate each new column of the table. The procedure is illustrated on several test sequences including one drawn from recent research in statistical mechanics. The results of these applications are very encouraging and suggest that the method should be a useful addition to algorithms for accelerating logarithmically convergent sequences (for example, see [5]). 


\section{Acknowledgement}

One of us (MNB) would like to thank Professor C. Brezinski for useful correspondence.

\section{References}

[1] C. Brezinski, “Accélération de suites à convergence logarithmique", C. R. Acad. Sci. Paris Sér. A-B 273 (1971), A727-A730.

[2] C. Brezinski, Accélération de la convergence en analyse numérique, Lecture Notes in Mathematics, vol. 584 (Springer-Verlag, Berlin, 1977).

[3] F. Cordellier, "Caracterisation des suites que la première étape du $\theta$-algorithme transformé en suites constants", C. R. Acad. Sci. Paris Sér. A-B 284 (1977), A389-A392.

[4] J. E. Drummond, "Summing a common type of slowly convergent series of positive terms", $J$. Austral. Math. Soc. Ser. B 19 (1976), 416-421.

[5] C. J. Hamer and M. N. Barber, "Finite-lattice extrapolations for $Z_{3}$ and $Z_{5}$ models", J. Phys. A 14 (1981), 2009-2025.

[6] D. Levin, "Development of non-linear transformations for improving the convergence of sequences", Internat. J. Comput. Math. Ser. B 3 (1973), 371-388.

[7] S. Lubkin, "A method of summing infinite series", J. Res. Nat. Bur. Standards 48 (1952), $228-254$.

[8] B. Nienhuis, E. K. Riedel and M. Schick, "Magnetic exponents of the two-dimensional q-state Potts model", J. Phys. A 13 (1980), L189-L192.

[9] A. Ralston, $A$ first course in numerical anabysis (McGraw-Hill, New York, 1965).

[10] D. Shanks, "Non-linear transformations of divergent and slowly convergent sequences", $J$. Math. and Phys. 34 (1955), 1-42.

[11] D. A. Smith and W. F. Ford, "Acceleration of linear and logarithmic convergence", SIAM J. Numer. Anal. 16 (1979), 223-240.

[12] J.-M. Vanden Broeck and L. W. Schwartz, "A one-parameter family of sequence transformations", SIAM J. Math. Anal. 10 (1979), 658-666.

[13] P. Wynn, "Upon systems of recursions which obtain among the quotients of the Pade table", Numer. Math. 8 (1966), 264-269.

Department of Applied Mathematics

University of New South Wales

P. O. Box 1

Kensington

N.S.W. 2033

and

Department of Theoretical Physics

Research School of Physical Sciences

Australian National University

Canberra

A.C.T. 2600 